\title{
Desenvolvimento sustentável e turismo indígena: uma análise das oportunidades e limitações do turismo nas comunidades indígenas da Reserva São Marcos (RR)
}

\author{
Indigenous tourism and sustainable development: an analysis of \\ opportunities and limitations of tourism in indigenous communities of \\ São Marcos Reserve (RR, Brazil)
}

\section{Cristiane Nascimento Brandão, José Carlos Barbieri, Edgar Reyes Junior}

\begin{abstract}
RESUMO
Recentemente, o interesse pelo turismo indígena aumentou significativamente no Brasil. Pesquisas internacionais centram suas discussões em torno da autonomia e sustentabilidade dos povos indígenas, do papel da sua cultura como produto turístico e dos problemas associados às políticas públicas nacionais. No Brasil, o turismo indígena não é um segmento reconhecido pelo poder público, embora estejam em curso diversas iniciativas conduzidas por comunidades indígenas. $\mathrm{O}$ objetivo deste artigo é apresentar os resultados de uma pesquisa exploratória para analisar as perspectivas dos povos indígenas da Reserva São Marcos (RR), quanto ao desenvolvimento do turismo indígena e sua influência na sustentabilidade ambiental, econômica e social. Foram realizadas entrevistas com lideranças e representantes do órgão responsável pela execução da política indigenista no Brasil, a FUNAI. Posteriormente, foi aplicado um questionário em 64 lideranças indígenas representes de 32 aldeias. O estudo revelou que essas lideranças consideram o turismo uma oportunidade para conquistar autonomia, controle e sustentabilidade econômica, social, cultural e ambiental, mas apontam diversos problemas que podem trazer para as áreas indígenas e suas comunidades, como conflitos entre membros da comunidade, distribuição dos benefícios, perda da identidade cultural, perturbação da vida selvagem, ameaça a espécies da região e poluição de lagos e rios da reserva.
\end{abstract}

PALAVRAS-CHAVE: Desenvolvimento Sustentável; Turismo Sustentável; Turismo Indígena. 
Desenvolvimento sustentável e turismo indígena: uma análise das oportunidades e limitações do turismo nas comunidades indígenas da Reserva São Marcos (RR)

\begin{abstract}
Recently, the interest in indigenous tourism has increased significantly in Brazil. International researchers focus their discussions around sustainability and autonomy of indigenous people, the role of culture as a tourism product and the problems related to national public policies. In Brazil, Indigenous tourism is not recognized by the government, although there are several ongoing initiatives led by indigenous communities. This paper aims to present the results of a survey that analyzed the perspectives of indigenous people of São Marcos Reserve (RR, Brazil), concerning the development of indigenous tourism and its influence on environmental, economic and social sustainability. Interviews were conducted with leaders and representatives of the implementing agency of indigenous policy in Brazil, FUNAI. Afterwards, a questionnaire was administered in 64 indigenous leaderships from 32 villages. The study revealed that these leaders consider tourism as an opportunity to get autonomy, control and economic, social, cultural and environmental sustainability. However the results also show several problems caused by tourism to the communities and indigenous areas, such as conflicts between community members, distribution of benefits, loss of cultural identity, disturbance of wildlife, threat to the region species and pollution of rivers and lakes of the reserve.
\end{abstract}

KEYWORDS: Sustainable Development; Sustainable Tourism; Indigenous Tourism.

\title{
Introdução
}

O interesse em torno do desenvolvimento de turismo indígena tem aumentado significativamente (NEPAL, 2005). Segundo o autor, as discussões estão centradas principalmente nos aspectos de capacitação, autonomia e sustentabilidade. Ryan (2000 apud NEPAL, 2005) também menciona os povos indígenas, o seu papel na indústria do turismo, a natureza de sua cultura como produto turístico e os problemas associados à regulamentação, como os principais tópicos dentro da literatura do turismo. No Brasil, o turismo indígena é pouco difundido, ainda não é um segmento reconhecido pelo poder público e tampouco possui regulamentação específica. Apesar de várias iniciativas existentes, poucas se tornam temas de estudos científicos.

O presente artigo tem como objetivo analisar as perspectivas dos povos indígenas da Reserva São Marcos, localizada no Estado de Roraima, quanto ao desenvolvimento do turismo indígena e sua influência na sustentabilidade ambiental, econômica e social. Trata-se de um estudo exploratório baseado na análise de dados primários (entrevistas com lideranças indígenas e agentes públicos relacionados com a política indigenista nacional, e questionários aplicados em 64 lideranças indígenas de 32 aldeias) e secundários (documentos, relatórios e material publicado). Para a coleta, foi utilizado questionário adaptado de Nepal (2005). A escolha da Terra Indígena (TI) São Marcos para a aplicação deste estudo se deu em função das iniciativas de algumas aldeias pertencentes a TI em inserir o turismo como atividade econômica. Algumas dessas comunidades têm trabalhado no turismo há pelo menos 10 anos sem qualquer 
apoio do poder público estadual ou federal. Tais iniciativas chamaram atenção de outras lideranças indígenas que passaram a se mobilizar para aderir ao turismo. Atualmente, líderes de comunidades se organizam para realizar consultas públicas pela regulamentação da atividade.

Este trabalho pretende contribuir para preencher uma lacuna do campo de estudo sobre turismo sustentável nas terras Indígenas de um modo geral e da Amazônia Brasileira de maneira específica. Assim em primeiro lugar, será apresentada uma discussão sobre desenvolvimento sustentável e turismo sustentável, de onde surgem os conceitos relacionados com o turismo em terras indígenas. Este tipo de turismo é pouco explorado na literatura nacional, seja porque há poucas experiências concretas em andamento, seja porque os assuntos relacionados com as comunidades indígenas têm sido tratados sob a ótica do Direito e da Antropologia dentro de enfoques de políticas públicas indigenistas, uma esfera de competência da União, como estabelece a Constituição da República Federativa do Brasil de 1.988. Por isso, os profissionais que lidam com o turismo pouco têm se aventurado nessa área, e da mesma forma, os pesquisadores e acadêmicos. Por isso, a seção referente ao turismo em áreas indígenas tem como base uma literatura desenvolvida em outros países. É notória a escassez de obras científicas sobre este tema no Brasil, como se pode observar na revisão teórica. Após a revisão teórica, será apresentado o método de pesquisa adotado para estudar o turismo na reserva indígena de São Marcos - Estado de Roraima. As últimas seções apresentam e discutem os resultados da pesquisa, bem como suas limitações, e fazem recomendações para estudos futuros.

\section{Revisão bibliográfica}

As ideias sobre desenvolvimento sustentável têm suas origens na busca de soluções para as crises sociais e ambientais que se tornam cada vez mais profundas e adquirem uma dimensão planetária. Entre as primeiras manifestações estão os estudos da UNESCO e da União Internacional para a Conservação da Natureza (IUCN) que viriam a gerar o programa Homem e Biosfera na década de 1970 com o objetivo de criar as bases científicas para melhorar as relações dos seres humanos com 0 meio ambiente. Esse programa, que existe até hoje, tem entre suas atividades mais conhecidas a criação de uma rede de reservas da biosfera. A Conferência das Nações Unidas sobre o Meio Ambiente Humano, realizada em Estocolmo em 1972, dando continuidade aos estudos dessas organizações, enfatizou a necessidade de vincular o desenvolvimento socioeconômico ao cuidado com o meio ambiente, este considerado como o provedor daquele, como expresso em um dos princípios aprovados:

os recursos naturais da terra incluídos o ar, a água, a terra, a flora e a fauna e especialmente amostras representativas dos ecossistemas naturais devem ser preservados em benefício das gerações presentes e futuras, mediante uma cuidadosa planificação ou ordenamento (Princípio 2).

Esse entendimento foi aprofundado pela Comissão Mundial sobre Meio Ambi- 
Desenvolvimento sustentável e turismo indígena: uma análise das oportunidades e limitações do turismo nas comunidades indígenas da Reserva São Marcos (RR)

ente e Desenvolvimento (CMMAD), conhecida como Comissão Brundtland (de Gro Harlen Brundtald, ex Primeira Ministra da Noruega). Em seu famoso relatório, denominado Nosso Futuro Comum, esta Comissão apresenta a definição mais divulgada sobre desenvolvimento sustentável:

desenvolvimento sustentável é aquele que atende às necessidades do presente sem comprometer a possibilidade das gerações futuras de atenderem às suas próprias necessidades (CMMAD, 1991; pg. 46).

Sobre o debate a respeito de crescimento e desenvolvimento, algo que sempre vem a tona, a CMMAD defende a ideia de retomada do crescimento econômico como condição fundamental para erradicar a pobreza, porém aponta para a necessidade de modificar a qualidade do crescimento para torná-lo mais justo, equitativo e menos intensivo em materiais e energia. A partir da publicação desse relatório e da realização da Conferência das Nações Unidas sobre Meio Ambiente e Desenvolvimento no Rio de Janeiro em 1992, esse modo de conceber o desenvolvimento ganha adeptos no mundo todo de forma crescente, sendo um tema considerado com muita frequência em políticas públicas e empresariais, embora esteja longe de ser um consenso, como mostram Gladwin, Kennelly e Krause (1995), Alier e Jusmet (2000), Costanza (2001), Nobre (2002), Fergus e Rowney (2005), Barbieri e Cajazeira (2009), entre outros.

Para Whitford e Ruhanen (2010) o apoio dado ao conceito tem sido atribuído à semântica atraente do termo, que oferece, embora de maneira contraditória, a possibilidade de dar continuidade ao desenvolvimento econômico que não prejudique a capacidade de carga do planeta. Para esses autores, o desenvolvimento sustentável implica em: 1) ambientes construídos a partir da reutilização de materiais e da utilização sustentável dos recursos naturais; 2) ecossistemas mais saudáveis; 3) desenvolvimento econômico mais sensível às necessidades do lugar, em vez de promover os lucros a uma minoria; 3 ) melhoria e distribuição equitativa das condições ambientais e econômicas.

O desenvolvimento sustentável como colocado acima remete ao plano global, é projeto para toda a Humanidade, o que traz um complicador a mais quando se pensa em sua operacionalização. Se já é difícil implementar políticas de desenvolvimento nacional subnacional e local, como ficou fartamente mostrado nas experiências de vários países, dentre eles o Brasil, na escala planetária espera-se encontrar maiores dificuldades. Por isso, a segmentação por atividades ou por setores tem sido uma das formas de superar as dificuldades, resultando as propostas sobre agricultura sustentável, indústria sustentável, cidades sustentáveis, energia sustentável, construções sustentáveis, logística sustentável e, como não poderia ficar fora, turismo sustentável. A palavra sustentável, um adjetivo que modifica o substantivo, traz a ideia de um novo modo de pensar e praticar as atividades humanas de um modo geral para que elas contribuam para alcançar o desenvolvimento sustentável na sua área de influência. 


\section{Turismo Sustentável}

Turismo sustentável é um modo de conceber o turismo em qualquer uma das suas modalidades, independentemente do local em que é praticado. É um termo mais amplo aplicável a qualquer modalidade de turismo e não apenas aquelas que têm na natureza o seu elemento de atração, como o ecoturismo e o turismo de natureza. Vários pesquisadores ampliaram o significado de turismo para incluir os conceitos e objetivos do desenvolvimento sustentável, o que implica na concepção de uma atividade com viabilidade em longo prazo e boa qualidade dos recursos naturais e humanos (BRAMWELL; LANE, 1993). Questões de sustentabilidade tornaram-se parte essencial do tecido social e político na maioria dos países, e o envolvimento de todos os intervenientes, incluindo as comunidades, também foi amplamente discutido (JAYAWARDENA et al., 2008).

Como mostra Swarbrooke (2000), os debates a respeito de turismo sustentável começaram na década de 1960 com a explosão do turismo em massa e seus impactos negativos. Na década seguinte observou-se um aumento da preocupação sobre os impactos negativos do turismo. Na década de 1980, com a preocupação e reconhecimento dos impactos negativos do turismo, começou a ser usada a expressão "turismo sustentável", levando em consideração as implicações do relatório da CMMAD, mencionado na seção anterior. A partir da década de 1990 a expressão "turismo sustentável" tornou-se frequente, acompanhando a popularidade do conceito de desenvolvimento sustentável, como já mostrado.

Hunter e Green (1995) e Organização Mundial do Turismo (WTO, 2004) sugerem que a sustentabilidade do turismo inclui qualidade de vida das comunidades de acolhimento, a satisfação do visitante e uso adequado dos recursos naturais e sociais. Nesse sentido, a WTO descreve o desenvolvimento do turismo sustentável como um processo que atende às necessidades atuais dos turistas e das comunidades receptoras, sem comprometer a capacidade de atender as necessidades das gerações futuras (WTO, 2004). Esta definição é a mesma da CMMAD, apenas introduzindo a palavra turismo. Nessa mesma linha, Swarbrooke (2000, p.19) o define turismo sustentável como "turismo que é economicamente viável, mas que não destrói os recursos dos quais o turismo do futuro dependerá, principalmente o meio físico e o tecido social da comunidade local'.

O objetivo do turismo sustentável é satisfazer as necessidades básicas da sociedade e ampliar a oportunidade para uma maior qualidade de vida (WTO, 2005). Para atingir este objetivo, Byrd (2007) entende que deve haver uma estrutura social que facilite a resolução de conflitos. Para esse autor, o sistema econômico deve ser capaz de produzir uma fonte contínua de excedentes e uma fonte de conhecimento técnico. A preocupação com a conservação do meio ambiente deve ser permanente, pois não se trata de processos estáticos, mas dinâmicos e, portanto, flexíveis e adaptáveis.

O turismo pode gerar impactos positivos e negativos no local de recepção, dependendo de como é planejado, implementado e gerenciado. Swarbrooke (2000) mostra que são necessárias condições favoráveis para que o turismo contribua para o 
Desenvolvimento sustentável e turismo indígena: uma análise das oportunidades e limitações do turismo nas comunidades indígenas da Reserva São Marcos (RR)

desenvolvimento econômico e social dentro das capacidades de carga dos ecossistemas. Dessa maneira, o turismo sustentável requer a participação de todos os interessados (cidadãos, empresários, líderes comunitários, entre outros), bem como de lideranças políticas para garantir consenso nas decisões, conforme mencionam Butts e Singh (2010). O foco na participação das partes interessadas realça ainda mais a sua capacidade de lidar com vários problemas percebidos como apontaram WTO (2004), Byrd (2007), Timur e Getz (2009) e Nizic et al. (2010). Para esses últimos autores, o turismo sustentável é o turismo com base em um acordo com a comunidade local, empresários e outros. Segundo eles, há no momento uma tendência para desenvolver o turismo de uma forma justa e aceitável para as comunidades locais, economicamente sustentável ao longo do tempo e que evita atrações turísticas que causem prejuísos ao ambiente físico.

Nizic et al. (2010) afirmam que é necessário ter uma estrutura de gestão com metas claras e coerentes, determinadas em conjunto por todas as partes interessadas e com base na realidade das localidades e das regiões (NIZIC et al., 2010). Usar da melhor maneira possível os recursos disponíveis é um dos principais desafios do turismo sustentável, pois, conforme mencionam Johnston e Tyrrel (2007), os objetivos da comunidade de acolhimento com relação ao turismo muitas vezes diferem daqueles da indústria do turismo, ou seja, as soluções mais desejáveis para a indústria do turismo podem não ser desejáveis para os residentes locais. Dessa forma, os processos de gestão dos destinos turísticos devem ser baseados em um conceito que permita a identificação de soluções eficientes e eficazes, sobre uma estrutura holística que inclua política, planejamento e desenvolvimento de forma a garantir que os benefícios do turismo sejam distribuídos equitativamente entre todas as partes interessadas (DOUGLAS, 2006; RUHANEN, 2006; LIM; COOPER, 2009).

\section{Turismo em Terras Indígenas}

Hinch e Butler (1996, p. 9) definem o turismo indígena como "atividade turística em que os povos indígenas estão diretamente envolvidos, quer através do controle ou por verem a sua cultura servir como a essência da atração". Conforme Parker (1993, apud ZEPPEL, 2006), na Austrália, turismo indígena ou aborígene é aquele em que o produto turístico é de propriedade indígena, emprega ou é operado pelos povos indígenas. No Canadá o turismo indígena é reconhecido como qualquer produto ou serviço de turismo operado por eles em suas reservas.

Segundo Smith (2006) turismo indígena é a atividade que atrai turistas para fazer visitas às aldeias indígenas, a observação de danças e cerimônias, compras de artesanato, dentre outras curiosidades. Para Hitchcock (1999, p. 18) "turismo indígena se caracteriza na busca pelo exótico, intocado, em sua forma autêntica". Já nos trabalhos de Bolnick (2003), o etnoturismo envolve a exibição da cultura e do estilo de vida indígena para os visitantes, por meio de interações pessoais, facilitando a comunicação intercultural e a educação. Para Zeppel (1999, apud RYAN; HUYTON, 2005), o produto do turismo indígena tem as seguintes características: (1) os próprios povos indígenas; (2) as práticas da cultura indígena; (3) o artesanato indígena; e (4) a gastronomia indígena, dentre outros atrativos. Como resultado, constrói-se uma maior 
compreensão e tolerância entre diferentes culturas. Como diz Weaver (2010), cada etnia possui uma cultura própria e adaptada às suas necessidades, organizadas em um contexto específico onde a natureza é fundamental para o desenvolvimento de seu modo de vida.

Para Pitchford (2006) o turismo indígena, por ter suas raízes na antropologia, muitas vezes é tido como um subconjunto do turismo cultural ou do etnoturismo. Já para Sinclair (2003), este subcampo é visto, mais claramente, como um fenômeno interdisciplinar dentro do campo de estudo do turismo, e o considera uma subdivisão do ecoturismo. Conforme Hinch e Butler (1996) o turismo indígena surgiu como um campo da investigação científica a partir da década de 1970, com trabalhos de investigação antropológica. Posteriormente, na década de 1990, o campo foi inserido no contexto de desenvolvimento econômico estratégico, que aperfeiçoou e analisou a relação contenciosa entre comunidades indígenas, áreas protegidas e ecoturismo (SMITH, 1989 apud HINCH; BUTLER, 1996). Foi durante estas duas fases que questões relevantes do turismo indígena começaram a ser moldadas de forma mais explícita dentro de um contexto de sustentabilidade.

De acordo com Brandão et a.I (2011), em pesquisa recente, analisaram 45 artigos no período de 2005 a 2010, publicados em seis revistas intenacionais dedicadas ao turismo e identificaram que os temas mais abordados foram o desenvolvimento sustentável e turismo sustentável, o que demonstra que o tema continua relevante e atual. Observou-se que quando se fala em turismo indígena, a temática sustetabilidade está presente na maioria das vezes. Entre os temas tratados nos artigos, os mais frequentes foram: desenvolvimento sustentável, turismo sustentável em áreas protegidas, parcerias e políticas públicas.

Weaver (2009) menciona que quase todos os povos indígenas já foram afetados em maior ou menor grau pela difusão do turismo de massa, nos EUA, na Austrália e no Canadá. Este tipo de turismo nem sempre está apoiado nos princípios da sustentabilidade. Dessa forma, esse autor recomenda que as questões pertinentes ao turismo devam ser moldadas em torno de um contexto de sustentabilidade. Conforme já dizia Swarbrooke (2000), o modo de vida dos povos indígenas pode contribuir para a conservação do meio ambiente, em especial a conservação da biodiversidade. A Agenda 21, aprovada na Conferência das Nações Unidas sobre Meio Ambiente e Desenvolvimento em 1992, reconhece explicitamente esse fato ao considerar os povos indígenas como parceiros principais para a promoção do desenvolvimento sustentável, para os quais dedica o Capítulo 26. A Declaração sobre o Desenvolvimento e Meio Ambiente, também aprovada nessa Conferência, estabelece que:

os povos indígenas e suas comunidades, bem como outras comunidades locais, desempenham um papel fundamental na gestão e no desenvolvimento do meio ambiente, em função de seus conhecimentos e suas práticas tradicionais. Os Estados devem reconhecer e dar apoio devido a sua identidade, cultura e interesses, e assegurar sua participação efetiva no processo de busca do desenvolvimento sustentável (Princípio 22). 
Desenvolvimento sustentável e turismo indígena: uma análise das oportunidades e limitações do turismo nas comunidades indígenas da Reserva São Marcos (RR)

\section{Legislação indigenista}

Souza e Barbosa (2011) mostram que a legislação indígena seguiu ao longo da história o paradigma do extermínio, o da integração e, por último, o do reconhecimento de direitos originários e ampliação de garantias com a promulgação da Constituição da República Federativa do Brasil de 1988. De acordo com Marés (2001), no período colonial o colonizador declarou guerra aos indígenas, fazendo deles escravos e a legislação procurou integrá-los, por meio do casamento, da catequese ou pela integração como trabalhadores livres. Após a independência, os índios sequer foram citados nas Constituições de 1824 e 1891. A política indigenista efetivamente começa em 1910 quando foi criado o Serviço de Proteção ao Índio, cuja vigência termina em 1967 ao ser substituído pela Fundação Nacional do Índio (FUNAI), em vigor até os dias atuais (OLIVEIRA; FREIRE, 2006).

A Constituição Federal de 1988 (CF/88), da mesma forma que as anteriores, também estabelece a competência privativa da União para legislar sobre as populações indígenas. Além de disposições esparsas sobre estas populações, a CF/88 dedica a elas o Título VIII,Capítulo VIII, que trata da ordem social. Ao indígena é reconhecida sua organização social, costumes, línguas, crenças e tradições, e os direitos originários sobre as terras que tradicionalmente ocupam, competindo à União demarcá-las, proteger e fazer respeitar todos os seus bens (BRASIL, 1988, art. 231). O Estatuto do Índio, instituído pela Lei no 6.001 de 1.973, por não conter dispositivos contrários a ela, foi recepcionada pela $\mathrm{CF} / 88$.

De acordo com Marés (2001), foi a CF/88 que reconheceu o direito dos indígenas de continuarem a serem índios sem a necessidade de integração na sociedade nacional. A CF/88 garante o uso das terras habitadas pelos índios, assim como, sua utilização para atividades produtivas, de forma a promover seu bem estar. Nesse contexto, assim como a agricultura, a caça e a pesca, o turismo poderia estar incluído. Ela não proíbe, em momento algum, o exercício do turismo como atividade econômica em terras indígenas.

A Política Nacional de Gestão Territorial e Ambiental de Terras Indígenas (PNGATI,), instituída pelo Decreto 7.747 de 2.012, tem por objetivo garantir e promover a proteção, a recuperação, a conservação e o uso sustentável dos recursos naturais das terras e territórios indígenas, assegurando a integridade do patrimônio indígena, a melhoria da qualidade de vida e as condições plenas de reprodução física e cultural das atuais e futuras gerações dos povos indígenas. A PNGATI estabelece objetivos específicos estruturados em sete eixos, sendo que o eixo 5 refere-se ao uso sustentável de recursos naturais e iniciativas produtivas indígenas. Entre os objetivos específicos desse eixo está o apoio às iniciativas indígenas sustentáveis de etnoturismo e de ecoturismo, respeitada a decisão da comunidade e a diversidade dos povos indígenas, promovendo-se, quando couber, estudos prévios, diagnósticos de impacto socioambientais e a capacitação das comunidades indígenas para a gestão dessas atividades (art. 4ํㅜㄴ, inciso V, g).

A PNGATI fala em etnoturismo e ecoturismo. Grosso modo, etnoturismo pode ser definido como turismo voltado para viagens a fim de colocar os turistas em conta- 
to com grupos étnicos no intuito de que aqueles possam conhecer seus usos e costumes. Essa modalidade de turismo também é consistente com o ecoturismo, basta ver quantas vezes a legislação indígena fala em meio ambiente, como mostram Brandão et al. (2011). Ceballos-Lascuráin (1999), a quem se atribui o uso pioneiro da palavra ecoturismo, entende que esta modalidade envolve viagem a áreas relativamente preservadas com objetivo específico de lazer, de estudo ou para admirar paisagens, fauna e flora, assim como qualquer manifestação cultural existente. Observa-se, portanto, que não há qualquer vedação legal no que se refere à prática de atividades econômicas no território indígena. O turismo pode ser uma opção que, além de possibilitar um incremento na renda, pode ainda promover o resgate e a valorização da cultura indígena, bem como, a preservação do meio ambiente, tendo em vista que cultura e meio ambiente são os principais elementos do turismo indígena.

\section{As Terras Indígenas São Marcos (RR)}

O objetivo deste artigo é analisar as perspectivas dos povos indígenas da Reserva São Marcos (RR) quanto ao desenvolvimento do turismo indígena e sua influência na sustentabilidade ambiental, econômica e social. Essa reserva está situada ao norte do Estado de Roraima, a Terra Indígena São Marcos é a maior em extensão de terras já demarcadas em área de lavrado e a única cortada por rodovia federal asfaltada, e possui uma extensão total de 654.110 hectares. A criação desta Reserva indígena remonta à história da colonização do Rio Branco pelos portugueses e coincide com a introdução da pecuária nos campos naturais da região (FUNAl, 2003).

No final do século XVIII foi criada a fazenda São Marcos, cujos limites descritos pelo Ministério da Fazenda, coincidem precisamente aos da atual Terra Indígena de São Marcos. Consideradas latifúndios de propriedade da União e de importância geopolítica por estarem situadas em fronteiras em litígio, essas áreas foram entregues ao Serviço de Proteção ao Índio (SPI), que posteriormente instalou um posto indígena em sua sede. A área de atuação deste órgão consistia no atendimento às populações indígenas, principalmente nas questões referentes às atividades agropecuárias. Mais tarde, o órgão foi extinto com a criação da Fundação Nacional do Índio (FUNAI). A Terra Indígena São Marcos foi demarcada em 1976 e, homologada pelo Decreto Presidencial no 312 de 29/10/1991 (RORAIMA, 2010). Dentro dos limites da Terra Indígena de São Marcos habitam três etnias indígenas: Macuxi e Taurepang, de filiação linguística Caribe; e Wapixana, de filiação Aruak. A população Taurepang, a menos numerosa, concentra-se na região norte da Terra Indigna de São Marcos; a Wapixana, na região sul; e a Macuxi, a mais numerosa, encontra-se dispersa por toda a área. Segundo levantamento realizado em 2003 pela FUNAl, a população residente na área é de 3.554 índios distribuídos em 32 aldeias.

A estrutura social acontece de maneira similar para as três etnias. As aldeias ficam dispersas ao longo do perímetro da reserva e geralmente ocupam margens de cursos d'água secundários. Existe uma concepção de livre acesso dos indivíduos ou grupos domésticos a terra e aos recursos naturais. As aldeias apresentam existência 
Desenvolvimento sustentável e turismo indígena: uma análise das oportunidades e limitações do turismo nas comunidades indígenas da Reserva São Marcos (RR)

curta e a formação permanente de novas aldeias é um traço fundamental da estrutura social destes povos.

Quanto à organização social, esta se baseia no parentesco bilateral e as aldeias têm autonomia política marcante. São lideradas por um "tuxaua" eleito pela comunidade. Não existem conflitos entre as três etnias e o índice de casamentos entre eles é alto. Quanto à economia, os indígenas de São Marcos vivem basicamente da agricultura e da pecuária de subsistência. Duas aldeias se destacam no desenvolvimento do turismo, atividade inserida pelos próprios indígenas há aproximadamente dez anos. Ao acompanharem os resultados positivos provenientes do turismo, outras lideranças começaram se organizar para introduzir o turismo como atividade econômica.

\section{Metodologia}

Para analisar as perspectivas dos povos indígenas da Reserva São MarcosRoraima quanto ao desenvolvimento do turismo indígena e sua influência na sustentabilidade ambiental, econômica e social, foi realizado um estudo exploratório baseado na análise de dados primários (entrevista aplicada a instituições indigenistas e uma coleta de dados aplicada às lideranças indígenas) e secundários (documentos, relatórios e material publicado). Para a coleta, foi utilizado questionário adaptado de Nepal (2005).

A primeira etapa foi realizada por meio de entrevistas semiestruturadas com dois públicos-alvo. O primeiro é constituído pelos gestores e técnicos do órgão indigenista, a Fundação Nacional do Índio - FUNAI, totalizando 10 entrevistados que receberam a denominação de F1 a F10. Com relação ao segundo público-alvo, realizaram -se entrevistas semiestruturadas com 17 lideranças indígenas, designadas de L1 a L17. Também foram analisados relatórios, projetos, materiais publicados e outros documentos oriundos das próprias comunidades e do órgão indigenista. Ressalta-se que as informações obtidas através desses documentos foram importantes, uma vez que puderam ser relacionadas ao material coletado por meio de entrevistas. O roteiro de entrevista utilizado era composto de 20 perguntas abertas, cujas informações foram confrontadas com as obtidas nos documentos analisados. As entrevistas tiveram entre 20 e 30 minutos de duração e foram realizadas entre fevereiro e março de 2012.

No segundo momento da pesquisa foi aplicado um questionário composto somente de questões fechadas, para 64 lideranças Indígenas, representantes das 32 aldeias (dois representantes por comunidade) presentes na Terra Indígena São Marcos. A realização dessa etapa aconteceu quando esses sujeitos estavam reunidos em assembleia, que são reuniões periódicas realizadas com o objetivo de planejar ações das aldeias indígenas de São Marcos, visando seu funcionamento. Na ocasião discutiu-se sobre a visão e entendimento dos indígenas acerca do desenvolvimento das comunidades por meio do turismo, e o objetivo do presente trabalho também foi apresentado.

O questionário adaptado de Nepal (2005) contém questões que envolvem as três dimensões da sustentabilidade: a econômica, a social e a ambiental, além do as- 
pecto cultural, tendo em vista que esta dimensão também pode ser afetada pelo turismo (HINCH; BUTLER, 1996; WEAVER, 2009). Nepal (2005) desenvolveu o questionário original num estudo exploratório em que buscava analisar o entendimento de uma comunidade tradicional sobre as oportunidades e limitações do ecoturismo. $O$ questionário foi aplicado em Tl'azt'en, uma sub-tribo do grupo Dene (Atabascanos), onde estão 47 terras indígenas, distribuídas por cerca de $6.560 \mathrm{Km}^{2}$ em um ambiente natural relativamente intocado no centro de British Columbia, Canadá (NEPAL, 2005). Como resultado da pesquisa, o autor mencionou que a comunidade possui um grande desejo em desenvolver o ecoturismo e tem uma visão positiva da atividade, porém parecem desconhecer seus desafios e limitações.

Nepal (2005) recomenda que o questionário seja aplicado face a face, de modo que se garanta o entendimento das questões e seja totalmente respondido. Nesse contexto, considerando que seria necessária a tradução do material - do inglês para o português - alguns cuidados quanto à equivalência de conteúdo do material original foram atendidos. O material traduzido passou por exaustivas revisões. Para haver mais clareza e confiabilidade no instrumento de coleta, foi realizado um pré-teste com 10 indígenas. O pré-teste revelou que o questionário necessitava de alguns ajustes de linguagem para facilitar o entendimento, o que posteriormente foi realizado. O questionário, composto de 48 questões, foi divido em três grupos, denominado de Grupo A, B e C. No Grupo A estão as questões relativas às perspectivas do desenvolvimento do turismo. As questões dos grupos $\mathrm{B}$ e $\mathrm{C}$ abordam temas pertinentes aos aspectos econômicos, sociais, culturais e ambientais: as do grupo B tratam das oportunidades decorrentes do turismo, e as do C, das limitações.

\section{Análise dos Resultados}

Os dados obtidos a partir do questionário fechado foram tabulados. O material coletado foi codificado e gerou um banco de dados, depois, foram calculadas as frequências das respostas, para isso utilizou-se o software Excel. A seguir será apresentada uma análise resumida das entrevistas; depois, a análise dos resultados da aplicação do questionário.

\section{A visão do órgão indigenista e das lideranças indígenas}

Existe uma forte interação dos povos indígenas com o meio ambiente (SWARBROKE, 2000). Dessa forma, é importante discutir a dimensão econômica, considerando suas relações com os aspectos ambientais. Assim, perguntou-se ao órgão indigenista se foi realizado algum estudo sobre os impactos das atividades econômicas realizadas pelas comunidades indígenas, inclusive o turismo. Obteve-se a seguinte resposta:

Recentemente foi realizado um estudo que analisou várias atividades, seus respectivos impactos e grau de severidade. Quanto ao turismo, não foi feito nenhum estudo, mas sabemos que também pode ocasionar impactos positivos e negativos (F2). 
Desenvolvimento sustentável e turismo indígena: uma análise das oportunidades e limitações do turismo nas comunidades indígenas da Reserva São Marcos (RR)

A Tabela 1, resumida de PTEI (2010), apresenta os impactos das atividades sobre os recursos naturais da TI São Marcos de que fala o entrevistado F2. Conforme $\mathrm{F} 2$, os estudos relatados abordam atividades tradicionais dos povos indígenas (agricultura, pastoreio e exploração de madeiras), sendo que a agricultura parece continuar como a principal forma subsistências dos povos indígenas. Não existe nenhum estudo sobre os impactos provenientes do turismo.

Tabela 1: Impactos ambientais das atividades tradicionais das comunidades.

Table 1: Environmental impacts of the activities of traditional communities.

\begin{tabular}{|l|l|l|}
\hline \multicolumn{1}{|c|}{ Atividades } & Grau de severida- & \multicolumn{1}{c|}{ Impacto } \\
\hline Roças tradicionais & Moderado & $\begin{array}{l}\text { Apesar das roças abrangerem anualmente áreas relativamente } \\
\text { pequenas, o fogo usado no preparo da terra pode escapar e } \\
\text { queimar áreas subjacentes. }\end{array}$ \\
\hline $\begin{array}{l}\text { Roças mecaniza- } \\
\text { das }\end{array}$ & Moderado & $\begin{array}{l}\text { Reduz o risco do fogo, mas atrasa a recuperação da vegetação } \\
\text { secundária. }\end{array}$ \\
\hline Fogo & Severo & Mesmo o fogo de baixa intensidade pode ocasionar a perda de \\
\hline $\begin{array}{l}\text { Extração de madei- } \\
\text { ra }\end{array}$ & Baixo & $\begin{array}{l}\text { A extração seletiva de madeiras em baixa intensidade deixa a } \\
\text { estrutura da floresta relativamente intacta. }\end{array}$ \\
\hline Venda de lenha & Moderado & $\begin{array}{l}\text { O comércio de lenha proveniente das derrubadas para a roça } \\
\text { tem estimulado o aumento do tamanho destas áreas para aque- }\end{array}$ \\
\hline
\end{tabular}

Fonte: Adaptado de Roraima PTEI, 2010.

Source: Adapted from Roraima PTEI, 2010.

Ao serem questionados sobre o que a FUNAI entende por desenvolvimento sustentável dos povos indígenas, obteve-se respostas controversas. Apesar de enfatizar que os índios têm autonomia para escolher suas atividades econômicas, por outro lado há o temor de que o aumento da renda possa causar desagregação e conflitos entre os membros das comunidades, conforme se pode ver pela fala de F3:

Para os povos indígenas, desenvolvimento sustentável é ter autonomia pra escolher as suas atividades econômicas, suas formas de se relacionar com a sociedade, com o meio ambiente, com a terra. Existe uma compreensão diferente para cada povo. A questão de ter uma renda, por exemplo, para os povos indígenas mais primitivos e isolados, falar em renda causa problemas sociais irreversíveis. Por isso é necessária reflexão, tanto nos programas sociais políticos, quanto nas atividades econômico-financeiras, para que não haja uma estratificação social, porque a sociedade indígena normalmente é organizada de maneira diferente da comunidade não indígena. Ela não é organizada por renda (F3).

Quando perguntados a respeito da regulamentação do turismo em Terras Indígenas, F9 respondeu o seguinte: 
Na realidade não existe lei alguma no Brasil que regulamente qualquer atividade comercial em áreas indígenas, nem mesmo o turismo. O que ocorre é a soberania da vontade dos indígenas. Eles escolhem com quais atividades vão trabalhar e nós (FUNAl) apenas aceitamos e apoiamos. Mas a nossa principal preocupação é se os indígenas estão realmente preparados para o turismo, e também que impactos essa atividade vai causar nas aldeias. Precisa ser feito um estudo para avaliar esses impactos. Outro ponto importante, é que não existe um planejamento para esta atividade, o que dificulta sua organização, gestão e divisão igualitária de renda (F9).

Note mais uma vez o temor do representante do órgão indigenista com os impactos do turismo e a ideia de que as comunidades ainda não estão preparadas para o turismo em suas áreas. Nos trabalhos de Douglas (2006); Ruhanen (2006) e Lim e Cooper (2009) são frequentes as recomendações quanto ao planejamento do turismo indígena que deve versar sobre uma estrutura holística que inclua política, planejamento e desenvolvimento de forma a garantir que os benefícios do turismo sejam distribuídos equitativamente entre todas as partes interessadas. Sobre isso, o respondente F10 afirma que

cabe aos governos federal e estadual trabalharem em uma política pública que regulamente não só o turismo, mas qualquer outra atividade econômica demandada pelos povos indígenas.

Depois, foi perguntado se a FUNAI acompanha/participa do turismo nas aldeias, o respondente $\mathrm{F} 5$ mencionou que

alguns técnicos já acompanharam turistas que estavam de visita na aldeia, em geral pequenos grupos de estudantes ou pesquisadores. Mas não é comum acontecer, pois não temos pessoal suficiente.

Perguntou-se às lideranças indígenas, qual a opinião sobre a introdução do turismo nas aldeias indígenas. Obtiveram-se as seguintes respostas de L1; L4, L5 e L6 respectivamente:

Os povos indígenas têm que ter autonomia sobre suas terras. Se a nossa aldeia quer o turismo, vamos fazer o turismo (L1).

O turismo pode ser uma coisa boa para nossos indígenas, porque vai trazer gente pra conhecer nossa cultura, pra comprar nosso artesanato e vai ter entrada de dinheiro e criação de emprego nas aldeias (L4).

Vejo que o turismo, com planejamento e participação de toda a comunidade pode trazer muitos benefícios pra nós. O povo indígena agora que está em paz com suas terras, quer trabalhar e desenvolver atividades que vão melhorar nosso modo de vida (L5). 
Desenvolvimento sustentável e turismo indígena: uma análise das oportunidades e limitações do turismo nas comunidades indígenas da Reserva São Marcos (RR)

As terras indígenas tem a cultura do povo, tem a natureza, nossa comida, nosso artesanato [...] as pessoas querem conhecer tudo isso. $\mathrm{E}$ o turismo vai melhorar muito nossa vida, porque vai gerar mais trabaIho para os jovens, para as mulheres e até para os velhos que tem muita história antiga pra contar. Turista também gosta de tomar banho na cachoeira, de caminhar na mata e ver os animais, tudo isso as aldeias tem pra mostrar (L6).

Pelas respostas percebe-se que as perspectivas acerca do turismo são positivas. E que a atividade pode proporcionar melhor qualidade de vida, emprego e renda, além de proporcionar mais autonomia aos povos indígenas. Nesse contexto, Scheyvens (1999) menciona que o turismo indígena pode ter seus impactos acompanhados e avaliados sob as pespectivas do poder econômico, da autonomia psicológica, do empoderamento social e da autonomia política. A resposta de L6 é coerênte com as observações de Zeppel (1999, apud RYAN; HUYTON, 2005), citadas anteriormente.

\section{A experiência da aldeia indígena Nova Esperança no turismo}

Segundo relatado pelo respondente L10, liderança da aldeia Nova Esperança, também pertencente a TI São Marcos, em 2000 as lideranças indígenas se reuniram para elaborar um planejamento de curto, médio e longo prazo para a aldeia. Assim, foi criada a ONG PRONESP - Programa de Desenvolvimento Sustentável de Nova Esperança. Ao realizar um diagnóstico da comunidade, verificaram-se vários aspectos positivos como a boa localização, o fato de a comunidade não apresentar vícios de paternalismo, o potencial para o ecoturismo, a existência de lagos perene que poderiam ser adaptados para a criação de peixes, para o lazer, dentre outros.

A comunidade decidiu investir no turismo, e no primeiro trimestre de 2001 participou de uma oficina de sensibilização realizada por consultor do PROECOTUR - Programa de Desenvolvimento do Ecoturismo na Amazônia Legal, do Ministério do Meio Ambiente. Posteriormente, participaram do $7^{\circ}$ edital do Programa de Pequenos Projetos (PPP-Ecos), gestado pelo ISPN-Instituto Sociedade, População e Natureza. O programa é financiado pelo Small Grants Programme (SGP), vinculado ao Fundo para o Meio Ambiente Global (GEF) da Organização das Nações Unidas. A execução dos projetos apoiados pelo PPP-ECOS se dá por meio do Programa das Nações Unidas para o Desenvolvimento (PNUD) com interveniência do Escritório de Serviços de Projetos das Nações Unidas (UNOPS). O PRONESP teve aprovado um projeto para realizar a capacitação dos moradores da comunidade para que os mesmos estivessem preparados para receber os turistas. De acordo com L10, Tuxaua da indígena Aldeia Nova Esperança, 
- PRONESP capacitou os moradores selecionados, realizou oficinas de artesanato, educação ambiental, gestão de resíduos e da água. Daí por diante a comunidade, ao longo dos últimos dez anos vem trabalhando no turismo. Atualmente, os moradores trabalham para melhorar a infraestrutura na aldeia, como a ampliação de restaurante, construção de pousada, melhorias no transporte e expansão das lojas de artesanatos. Os principais produtos turísticos da comunidade são as inúmeras trilhas ecológicas existentes, a principal delas é a Trilha do Coatá, que leva até um sítio arqueológico (L10).

Segundo as lideranças da aldeia indígena Nova Esperança o turismo proporcionou vários benefícios, levando a crer que o desenvolvimento da atividade foi uma escolha apropriada e bem trabalhada. Ainda segundo o Taxaua,

atualmente nós continuamos fortes com o turismo, mas não deixamos de lado a psicultura, nem a agricultura, pois entendemos que uma atividade complementa a outra. O que posso dizer, é que hoje a comunidade vive bem melhor do que antes do turismo. Se não fosse o turismo, nós teríamos investido em gado, e para a pecuária teríamos que desmatar uma grande área para fazer o pasto. Para o turismo não precisa desmatar nada, ao contrário, aprendemos a preservar o meio ambiente (L10).

Como afirma Lewis (2001) turismo é uma forma de implementar a sustentabilidade econômica, ambiental e social, já que a comunidade garante recursos e financiamentos; cria uma estrutura organizacional no local; e o turismo proporciona a articulação com empresas e instituições externas a comunidade.

\section{Análise dos resultados do questionário}

A Tabela 2 apresenta os resultados referentes ao Grupo A do questionário referente às perspectivas para o desenvolvimento do turismo. Como se vê, uma percentagem significativa dos respondentes se mostrou a favor do desenvolvimento do turismo em terras indígenas. Segundo as respostas, os indígenas acreditam que existe potencial em suas terras para desenvolver a atividade.

A maioria respondeu positivamente a todas as perguntas sobre o turismo. Eles acreditam que o turismo pode proporcionar valorização da cultura (73\%), pode melhorar o entendimento entre índios e não índios (82\%). Para os respondentes, o turismo pode melhorar as condições financeiras e sociais dos povos indígenas (84\%). Quanto aos efeitos negativos, por exemplo, aumento de drogas, álcool e prostituição, $45 \%$ afirmaram que o turismo não agravaria este tipo de problemas. Diferentemente da pesquisa realizada por Nepal (2005), os grupos indígenas do Canadá pareciam desconhecer seus desafios e limitações, conforme mencionado 
Desenvolvimento sustentável e turismo indígena: uma análise das oportunidades e limitações do turismo nas comunidades indígenas da Reserva São Marcos (RR)

anteriormente.

Tabela 2: Perspectivas para o desenvolvimento do turismo - Grupo A.

Table 2: Prospects for the development of tourism - Group A.

\begin{tabular}{|c|l|c|c|c|}
\hline \multicolumn{1}{|c|}{ Questões } & Sim & Não & Não sei \\
\hline 1 & Seria bom desenvolver o turismo em aldeias indígenas? & 61 & 1 & 0 \\
\hline 2 & As terras indígenas tem potencial turístico? & 53 & 3 & 6 \\
\hline 3 & $\begin{array}{l}\text { Você acredita que o turismo poderia proporcionar melhor entendi- } \\
\text { mento entre índios e não índios? }\end{array}$ & 51 & 5 & 6 \\
\hline 4 & $\begin{array}{l}\text { Você acredita que o turismo pode melhorar as condições financei- } \\
\text { ras e sociais dos povos indígenas? }\end{array}$ & 52 & 8 & 2 \\
\hline 5 & O turismo pode resultar em maior apreciação da cultura indígena? & 48 & 1 & 13 \\
\hline 6 & Você acredita que os indígenas irão valorizar ainda mais sua cultu- & 45 & 2 & 15 \\
\hline 7 & $\begin{array}{l}\text { Você acredita que o turismo pode trazer efeitos negativos aos po- } \\
\text { vos indígenas (ex: drogas, álcool, prostituição)? }\end{array}$ & 23 & 28 & 11 \\
\hline
\end{tabular}

Fonte: Pesquisa de campo (2012).

Source: Field survey (2012).

Com relação às questões do grupo $\mathrm{B}$ e $\mathrm{C}$ do questionário, em que foram abordados aspectos econômicos (questões de 1 a 5), sociais (6 a 11), culturais (12 a 16) e ambientais (17 a 21), observou-se que a maioria dos respondentes tem uma percepção positiva acerca da atividade, como mostra a Tabela 3. Com respeito ao aspecto econômico as respostas mais frequêntes foram: aumento de emprego, oportunidade de negócios, treinamento e desenvolvimento e oportunidades de negócio.

Já no aspecto social, questões como compreensão da cultura, aumento da autoestima, formação de parcerias, abertura para o diálogo e melhoria nas relações, foram as mais frequentes. Quanto aos aspectos culturais as respostas mais citadas foram: o aumento na consciência cultural, oportunidades de mostrar o artesanato indígena e promoção da cultura. Quanto ao aspecto ambiental, observou-se maior frequência nas seguintes respostas: conservação da fauna e flora, proteção do patrimônio natural e reforço dos valores de que fazemos parte da natureza.

A Tabela 4 mostra e frequência de respostas quanto as potenciais limitações do turismo indígena. As respostas relacionadas às limitações foram bem mais baixas se comparadas com o levantamento sobre as oportunidades (Tabela 3). O que reforça a ideia de que as lideranças indígenas apresentam uma percepção positiva quanto ao turismo. Com relação às limitações de natureza econômica, a frequência mais alta das respostas refere-se ao trabalho sazonal e mal remunerado (50\%). Vale mencionar que esse é um problema típico das atividades turísticas sujeitas às estações do ano, no caso do Estado de Roraima e praticamente toda a região Amazônica a estação das chuvas não é propícia ao turismo, de modo que nesta estação a visitação de turista reduza a quase zero, principalmente em função do acesso até as comunidades. 
Brandão, C.N.; Barbieri. J.C.; Reyes-Junior, E.

Tabela 3: Percepção das oportunidades do turismo - Grupo: B (Oportunidades).

Table 3: Perception of tourism opportunities - Group B (Opportunity).

\begin{tabular}{|c|c|c|c|}
\hline & ASPECTOS ECONÔMICOS & Freq. & $\%$ \\
\hline 1 & Aumento de postos de trabalho & 58 & 94 \\
\hline 2 & Oportunidades de negócios & 60 & 97 \\
\hline 3 & Desenvolvimento de infraestrutura & 61 & 98 \\
\hline 4 & Ferramenta de desenvolvimento e treinamento & 56 & 90 \\
\hline \multirow[t]{2}{*}{5} & Outro: & 2 & 3 \\
\hline & ASPECTOS SOCIAIS & & \\
\hline 6 & Compreensão compartilhada de culturas externas & 58 & 94 \\
\hline 7 & Aumento da autoestima & 62 & 100 \\
\hline 8 & Formação de parcerias & 54 & 87 \\
\hline 9 & Melhoria das relações com a comunidade de entorno & 56 & 90 \\
\hline 10 & Abertura para o diálogo e a comunicação & 55 & 89 \\
\hline \multirow[t]{2}{*}{11} & Outro: & 7 & 11 \\
\hline & ASPECTOS CULTURAIS & & \\
\hline 12 & Aumento da consciência cultural dos povos indígenas & 59 & 95 \\
\hline 13 & Oportunidade para mostrar artes e ofícios locais & 60 & 97 \\
\hline 14 & Valores tradicionais são apresentados aos visitantes & 60 & 97 \\
\hline 15 & Oportunidades para promoção da cultura & 57 & 92 \\
\hline \multirow[t]{2}{*}{16} & Outro: & 1 & 2 \\
\hline & ASPECTOS AMBIENTAIS & & \\
\hline 17 & Conservação da fauna e flora & 60 & 97 \\
\hline 18 & Proteção do patrimônio natural & 58 & 94 \\
\hline 19 & Sensibilização para a importância da vida selvagem & 55 & 89 \\
\hline 20 & Reforçar os valores de que fazemos parte da natureza & 61 & 98 \\
\hline 21 & Outro: & 3 & 5 \\
\hline
\end{tabular}

Fonte: Pesquisa de campo (2012).

Source: Field survey (2012).

Sobre os aspectos sociais, a resposta sobre conflitos entre os membros da comunidade foi a mais frequente, com $55 \%$. No aspecto cultural, o item "jovens tem vergonha da cultura indígena" totalizou 44\% das respostas. Enquanto que nos aspectos ambientais, as respostas mais frequentes foram "perturbação da vida selvagem" com $32 \%$ e "poluição de lagos e rios" com $29 \%$ das respostas. 
Desenvolvimento sustentável e turismo indígena: uma análise das oportunidades e limitações do turismo nas comunidades indígenas da Reserva São Marcos (RR)

Tabela 4: Percepção das limitações do turismo - Grupo C (Limitações).

Table 4: Perception of the limitations of tourism - Group C (Limitations).

\begin{tabular}{|c|l|c|c|}
\hline & ASPECTOS ECONÔMICOS & Freq. & $\%$ \\
\hline 1 & Poucas pessoas empregadas no turismo & 18 & 29 \\
\hline 2 & Trabalho sazonal e mal remunerado & 31 & 50 \\
\hline 3 & Empregos principalmente para pessoas de fora da comunidade indígena & 12 & 19 \\
\hline 4 & Pessoas influentes se beneficiarão mais & 25 & 40 \\
\hline 5 & Outro: & 9 & 15 \\
\hline & ASPECTOS SOCIAIS & & \\
\hline 6 & Conflitos entre os membros da comunidade & 34 & 55 \\
\hline 7 & Exclusão dos mais velhos & 23 & 37 \\
\hline 8 & Introdução de novos hábitos na comunidade & 24 & 39 \\
\hline 9 & Criação de diferentes classes sociais dentro da comunidade & 11 & 18 \\
\hline 10 & Outro: & 2 & 3 \\
\hline & ASPECTOS CULTURAIS & & \\
\hline 11 & Aceleração do processo de aculturação & 17 & 27 \\
\hline 12 & Jovens limitando estilo de vida dos turistas & 19 & 31 \\
\hline 13 & Jovens não respeitam os mais idosos & 16 & 26 \\
\hline 14 & Jovens tem vergonha da cultura & 27 & 44 \\
\hline 15 & Outro: & 5 & 8 \\
\hline & ASPECTOS AMBIENTAIS & & \\
\hline 16 & Perturbação da vida selvagem & 20 & 32 \\
\hline 17 & Poluição de lagos e rios & 18 & 29 \\
\hline 18 & Exploração de produtos florestais & 17 & 27 \\
\hline 19 & Fauna e flora ameaçada de extinção & 23 & 37 \\
\hline 20 & Outro: & 11 & 18 \\
\hline
\end{tabular}

Fonte: Pesquisa de campo (2012).

Source: Field survey (2012).

Comentando sobre as limitações do turismo nas terras indígenas, Nepal (2005), o autor que concebeu a base sobre a qual se adaptou o questionário para aplicação na pesquisa relatada, considera que o turismo deve ser cuidadosamente planejado e desenvolvido sobre as bases da sustentabilidade, a fim de garantir a longevidade dos projetos de turismo e evitar os impactos negativos. As respostas desse grupo de perguntas vão ao encontro das considerações de Nizic et al. (2010), Douglas (2006), Ruhanen (2006) e Lim e Cooper (2009) comentadas na revisão bibliográfica.

\section{Conclusão}

Este artigo apresenta os resultados de uma pesquisa que procurou analisar as perspectivas dos povos indígenas da Reserva São Marcos (RR), quanto ao desenvolvimento do turismo indígena e sua influência na sustentabilidade ambiental, econômica e social. As lideranças indígenas veem no turismo uma oportunidade para promover seu desenvolvimento e dessa forma conquistar autonomia, controle e sustentabilidade econômica, social, cultural e ambiental. Percebe-se com esse posicionamento a 
vontade de inovar por meio do turismo, e promover desenvolvimento dentro das reservas indígenas, evitando a migração para cidade. Porém, o mesmo não ocorre com os representantes do órgão indigenista responsável pela execução da política indigenista no Brasil, a FUNAI, que mantém uma posição de aceitar a vontade da comunidade ao mesmo tempo em que antevê problemas e considera que as comunidades não estão preparadas para essa atividade. Com as mudanças introduzidas pela CF/88, ao órgão público encarregado da política indigenista nacional cabe acatar as decisões das comunidades indígenas da Reserva São Marcos.

O desafio é desenvolver o turismo levando em conta os aspectos da sustentabilidade, a fim de minimizar as limitações e impactos. Para isso, é importante planejar e realizar o monitoramento da atividade, como foi amplamente recomendado por vários autores citados. O provimento de conhecimentos e instrumentos para tornar o turismo indígena sustentável é uma das atribuições do órgão indigenista nesta fase da história nacional inaugurada pela $\mathrm{CF} / 88$, como comentado na revisão bibliográfica. É importante salientar que não basta que as lideranças queiram introduzir o turismo nas aldeias, é necessária a aceitação, participação e cooperação de toda a comunidade indígena, a fim de garantir a qualidade do produto e os resultados positivos da atividade, bem como dos agentes locais relacionados com a indústria do turismo, como transportadores, agentes de viagens, provedores de alimentação e meios de hospedagens. Não ter considerado esses agentes é uma das limitações desse trabalho. Lembrando o que foi comentado na revisão bibliográfica, as melhores soluções para essa indústria podem não ser as mesmas para a comunidade de acolhimento dos turistas. Outra limitação foi restringir o estudo às comunidades de uma única Reserva. Para estudos futuros, recomenda-se aplicar a pesquisa em outras comunidades indígenas para verificar como outras aldeias indígenas da Região Amazônica enxergam o turismo em suas áreas.

\section{Referências bibliográficas}

ALIER, J.M; JUSMET, J.R. Economía ecológica y política ambiental. México, DF: PNUMA y Fondo de Cultura Econômica. 2000.

BARBIERI, J.C; CAJAZEIRA, J.E.R. Responsabilidade social empresarial e empresa sustentável. São Paulo, Saraiva, 2009.

BOLNICK, S. Promoting the culture sector through job creation and small enterprise development in SADC countries: the ethno-tourism industry. Geneva, International Labour Office, 2003.

BRAMWELL, W. e LANE, B. Sustainable tourism: an evolving global approach. Journal of Sustainable Tourism.v.1, n.1, p.1-5. 1993.

BRANDAO, C. N.; BARBIERI, J. C.; REYES JUNIOR, E. O Campo de Estudo do Turismo Sustentável em Comunidades Indígenas. In: XIII Anais do ENGEMA. São Paulo, 2011.

BRASIL. Constituição (1988). Constituição da República Federativa do Brasil. Brasília, DF: Senado, 1988.

BRASIL. Lei no 6.001/1973. Dispõe sobre o Estatuto do Índio. Diário Oficial da União. Brasília, DF, 21 de dezembro de 1973. 
Desenvolvimento sustentável e turismo indígena: uma análise das oportunidades e limitações do turismo nas comunidades indígenas da Reserva São Marcos (RR)

BRASIL. (PNGATI) Decreto no 7.747/2012. Institui a Política Nacional de Gestão Territorial e Ambiental de Terras Indígenas. Diário Oficial da União. Brasília, DF, 21 de junho de 2012

BUTTS, T.; SINGH, T.S Sustainable tourism as a tool for conservation and protection of the Amazon rainforest in Guyana? Worldwide Hospitality and Tourism Themes. v. 2, n. 2, pp. 173-185. 2010.

BYRD, E.T. Stakeholders in sustainable tourism development and their roles. Tourism Review, v. 62, n. 2. 2007.

CEBALLOS-LASCURÁIN, H. O ecoturismo como um fenômeno mundial. In: LINDBERG, K; HAWKINS,D.E. Ecoturismo: um guia para planejamento e gestão. São Paulo, Editora SENAC, 1999.

COMISSÃO MUNDIAL SOBRE MEIO AMBIENTE E DESENVOLVIMENTO. Nosso futuro comum. Rio de Janeiro: Fundação Getulio Vargas, 1991.

COSTANZA, R. Visions, values, valuation, and need for an ecological economics. BioScience, v. 51, n.6, p. 459-468. 2001.

DOUGLAS, C. H. Small island states and territories: sustainable development issues and strategies - challenges for changing islands in a changing world. Sustainable Development, v. 14, p.75-80. 2006.

FERGUS, A.H.T.; ROWNEY, J.I.A. Sustainable development: lost meaning and opportunity. Journal of Business Ethics, v. 60, p.17-27. 2005.

GLADWIN, T.N.; KENNELLY, J.J.; KRAUSE, T. Shifting paradigms for sustainable deveolpment: implications for management theory and research. Academy of Management Review, v. 20, n. 4, p. 874-907.1995.

HINCH, T.; BUTLER, R. Indigenous tourism: A common ground for discussion. London: International Thomson, Business Press, 1996.

HITCHCOCK, M. Tourism and ethnicity: situational perspectives. International Journal of Tourism Research, London, v. 1, p. 17-32.1999.

HUNTER, C; GREEN, H. Tourism and the environment: a sustainable relationship? London; New York: Routledge, 1995.

JAYAWARDENA, C. et al. Sustainable tourism development in Niagara Discussions, theories, projects and insights. International Journal of Contemporary Hospitality Management. v. 20, n. 3, p. 258-277. 2008.

JOHNSTON, J.; TYRREL, J. Management exercises and trainer's note in sustainable tourism and dynamics. International Journal of Culture, Tourism and Hospitality Research.v.1, n.4. 2007.

LEWIS, J. B. A rural tourism development model. Tourism Analysis, v.2, p. 91-105. 2001.

LIM, C. C. E COOPER, C. Beyond Sustainability: Optimising Island Tourism Development. International Journal of Tourism Research. v.11, p.89-103. 2009.

MARÉS, C.F.S.F. O renascer dos povos indígenas para o direito. Curitiba: Museu Nacional/Fundação Ford, 2001. 
Brandão, C.N.; Barbieri. J.C.; Reyes-Junior, E.

NEPAL, S.K. Limits to Indigenous Ecotourism: An exploratory analysis from the Tl'azt'en Territories, Northn British Columbia. In: RYAN, C.; AICKEN, M. (eds) Indigenous Tourism: The Commodification and Management of Culture.Oxford: London and New York, p.51-65. 2005.

NIZIC, M.K.; IVANOVIC, S.; DRPIC, D. Challenges to Sustainable Development In Island Tourism. South East European Journal of Economics and Business, 2010.

NOBRE, M. Crescimento econômico versus preservação ambiental: origens do conceito de desenvolvimento sustentável. In: NOBRE, M; AMAZONAS, M. C. Desenvolvimento sustentável: a institucionalização de um conceito. Brasília, IBAMA, 2002.

OLIVEIRA, J. P. O. ; FREIRE, C. A. R. A presença indígena na formação do Brasil. Brasília: Ministério da Educação, Secretaria de Educação Continuada, Alfabetização e Diversidade; LACED/Museu Nacional, 2006.

PITCHFORD, S. Identity tourism: A medium for Native American stories. Tourism Culture \& Communication. v.6, n. 2, p.85-105. 2006.

RYAN, C; HUYTON, J. Balanda tourists and aboriginal people (Chapter 5). Indigenous Tourist: the commodification and management of culture, ed. Elsevier: London and New York, pp. 51-65, 2005.

RORAIMA. Secretaria de Estado do Índio. Programa Territórios da Cidadania. Plano de Etnodesenvolvimento do Território do Território Indígena Raposa Serra do Sol e São Marcos - PTEI. Roraima, 2010. 222 p. (Relatório PTEI 2010).

RUHANEN, L. Sustainable tourism planning: An analysis of Queensland local tourism destinations. 2006. $447 \mathrm{f}$. PhD Thesis, Scholl of Tourism, University of Queensland, 2006.

SCHEYVENS, R. Ecotourism and the empowerment of local communities. Tourism Management, v. 20, p. 245-249. 1999.

SINCLAIR, D. Developing indigenous tourism: Challenges for the Guianas. International Journal of Contemporary Hospitality Management, v.15, n.3. 2003.

SMITH, T. Welfare, enterprise, and aboriginal community: the case of the western Australian Kimberley region, 1968-96. Australian Economic History Review, v. 46, n. 3, p. 242-267, nov. 2006.

SMITH, V. Hosts and guests: the Anthropology of Tourism.2.ed University of Pennsylvania Press: Philadelphia, 1998.

SOUZA, M. N.; BARBOSA, E. M. Direitos indígenas fundamentais e sua tutela na ordem jurídica brasileira. In: Âmbito Jurídico. Rio Grande, XIV, n. 85, fev 2011. Disponível em: HTTP://www.ambito-juridico.com.br/site/?

n link=revista artigos leitura id=8978\&revista caderno=9. Acesso em: 02 ago. 2012.

SWARBROOKE, J. Turismo sustentável: conceitos e impacto ambiental. São Paulo: Aleph, 2000.

TIMUR, S.; GETZ, D. Sustainable tourism development: how do destination stakeholders perceive sustainable urban tourism? Sustainable Development. v.17, p.220-232. 2009. 
WEAVER, D. Indigenous tourism stages and their implications for sustainability. Journal of Sustainable Tourism, v.18, n.1, p. 43-60. 2010.

WHITFORD, M.M.; RUHANEN, L. N. Australian indigenous tourism policy: practical and sustainable policies? Journal of Sustainable Tourism. v.18, n.4, p.475-496. 2010.

WTO - World Tourism Organization. Sustainable Tourism Development Guide for Local Planners. WTO: Madrid, Spain, 2004.

WTO. Making tourism more sustainable: a guide for policy makers/United Nations Environment Programme and World Tourism Organization. France; Spain. 2005.

ZEPPEL, H. Indigenous ecotourism: sustainable development and management. CABI. 2006.

Cristiane Nascimento Brandão: Fundação Getulio Vargas, São Paulo, SP, Brasil

Email: cristianne.brandao@gmail.com

Link para o currículo Lattes: http://lattes.cnpq.br/8182695538893409

José Carlos Barbieri: Fundação Getulio Vargas, São Paulo, SP, Brasil

Email: jose.barbieri@fgv.br

Link para o currículo Lattes: http://lattes.cnpq.br/0041377800166678

Edgar Reyes Junior: Universidade Federal de Roraima, Boa Vista, RR, Brasil

Email: edgarreyes2000@yahoo.com.br

Link para o currículo Lattes: http://lattes.cnpq.br/4600683588964302

Data de submissão: 20 de setembro de 2012

Data de recebimento de correções: 19 de novembro de 2012

Data do aceite: 19 de novembro de 2012

Avaliado anonimamente 